\title{
Slow variation in the Gompertz model
}

\author{
T. Grozdanovski* $\quad$ J. J. Shepherd ${ }^{\dagger}$
}

(Received 21 December 2005; revised 15 December 2006)

\begin{abstract}
In many differential, single species, population models, the intrinsic model parameters are assumed to be given constants. Typical examples are the "carrying capacity" and "rate constant" arising in the logistic model. Such constant parameters usually allow exact solution of the model equations, to completely describe the evolving population. However, this assumption of constancy is not realistic since such parameters may exhibit a wide range of variation with time. This investigation considers a particularly useful population modelthe Gompertz model - in the case where the model parameters vary slowly with time. Multi-timing methods construct an approximate expression for the population that has the advantages of being both explicit and giving results comparable to those obtained from numerical calculations.
\end{abstract}

*School of Mathematical and Geospatial Sciences, RMIT University, Melbourne, Australia. mailto:e61565@ems.rmit.edu.au

${ }^{\dagger}$ School of Mathematical and Geospatial Sciences, RMIT University, Melbourne, Australia. mailto: jshep@rmit.edu.au

See http://anziamj.austms.org.au/V47EMAC2005/Grozdanovski for this article, (C) Austral. Mathematical Soc. 2007. Published January 5, 2007. ISSN 1446-8735 


\section{Contents}

1 Introduction

C542

2 Multiscale formulation of the model

C544

3 Perturbation analysis

C545

4 Application to particular cases

C547

4.1 Exponentially varying carrying capacity . . . . . . . . .

C547

4.2 Periodically varying carrying capacity . . . . . . . . . .

C549

4.3 The bi-Gompertz model . . . . . . . . . . . . . . . C549

4.4 Periodically varying growth rate and carrying capacity . . C552

5 Discussion

C552

References

C554

\section{Introduction}

Whilst populations of individuals change in discrete steps, the evolution of populations are very well represented using the continuous tool of differential equations. When spatial effects such as diffusion and dispersion are neglected, the mathematical model of a single species population reduces to an initial value problem involving a single (usually nonlinear) ordinary differential equation in one variable. While such simplification may be criticised, such models still find application in the study of such disparate phenomena as the evolution of fish school populations, the reactions of chemical ions, or the spread of a single innovation or phenomenon. Moreover, such models often provide results that are of use in predicting behaviour.

Typically, such differential equation models contain parameters that de- 
fine the growth characteristics of the species involved, and its interaction with the microcosm. Usually, these parameters are taken as constants. This is often necessary to allow useful analysis of the model. However, such parameters usually do vary with time. In such cases, analysis of the model usually requires repetitive numerical simulations to enable some form of general statements to be made about the role played by these parameters in the population evolution.

Often, the model parameters vary slowly in time, due, perhaps, to seasonal variation. Then, the population variation may be viewed as depending on two time scales - the 'normal' intrinsic scale of the species, and the 'slow' time scale imposed by the parameter variation. In such cases, the model may be analysed using a "multi-time" approach, as applied, for example, in the area of mechanical oscillations [6].

In the present paper, we apply such analysis to a population $P(t, \varepsilon)$ given by the solution of the initial value problem

$$
\frac{d P(t, \varepsilon)}{d t}=r(\varepsilon t) P(t, \varepsilon) \ln \left(\frac{K(\varepsilon t)}{P(t, \varepsilon)}\right), \quad P(0, \varepsilon)=P_{0}>0 .
$$

In the above, $r$ and $K$ are given positive functions of their arguments, while $\varepsilon$ is a small positive parameter. Thus, $r(\varepsilon t)$ and $K(\varepsilon t)$ denote functions which vary slowly as $t$ varies, since an $O\left(\varepsilon^{-1}\right)$ change in $t$ is required to produce an $O(1)$ change in $r$ or $K$.

When $r$ and $K$ are constant, the above model reduces to the Gompertz model $[2,3]$, which has been used in modelling in such diverse areas as actuarial studies and economics, technology transfer and technology substitution, innovation diffusion, tumor growth including breast cancers, and a range of biological and botanical phenomena. Here, $r$ is the Malthusian growth constant, while $K$ is the carrying capacity, the limiting population supportable by the microcosm. Our model (1) above (also discussed in [4]) extends this to the situation where $r$ and $K$ vary slowly in time as a result of some background variation. 


\section{Multiscale formulation of the model}

The problem (1) depends on the two time scales $t$ and $\varepsilon t$. This implies that we should regard the solution $P(t, \varepsilon)$ as a function of these two time scales. This is the simpler approach adopted in earlier analysis of similar models [7]. However, it turns out that we need to propose more general time scales for (1), namely

$$
t_{0}=\frac{1}{\varepsilon} g\left(t_{1}\right) \quad \text { and } \quad t_{1}=\varepsilon t,
$$

where $g\left(t_{1}\right)$ is an arbitrary function of $t_{1}$ which is to be found. Here $t_{0}$ is a transformed "normal" time variable and $t_{1}$ is the "slow" time variable. Setting

$$
P(t, \varepsilon) \equiv p\left(t_{0}, t_{1}, \varepsilon\right),
$$

we get, on application of the chain rule,

$$
\frac{d P(t, \varepsilon)}{d t}=g^{\prime}\left(t_{1}\right) D_{0} p+\varepsilon D_{1} p,
$$

where $D_{0}$ and $D_{1}$ denote partial differential operators taken with respect to $t_{0}$ and $t_{1}$ respectively.

With these transformations, the multi-scaled Gompertz equation is

$$
g^{\prime}\left(t_{1}\right) D_{0} p+\varepsilon D_{1} p=r\left(t_{1}\right) p \ln \left(\frac{K\left(t_{1}\right)}{p}\right) .
$$

This equation (5) for $p$ now displays $\varepsilon$ explicitly, so that a perturbation approach based on $\varepsilon \rightarrow 0$ may be used. We also note that this is a partial differential equation. 


\section{Perturbation analysis}

We now express $p$ as a Poincaré expansion in $\varepsilon$,

$$
p\left(t_{0}, t_{1}, \varepsilon\right)=p_{0}\left(t_{0}, t_{1}\right)+\varepsilon p_{1}\left(t_{0}, t_{1}\right)+\varepsilon^{2} p_{2}\left(t_{0}, t_{1}\right)+\cdots,
$$

where this expansion is subject to the boundedness condition

$$
\left|\frac{p_{n+1}\left(t_{0}, t_{1}\right)}{p_{n}\left(t_{0}, t_{1}\right)}\right|<\infty, \quad n=0,1,2, \ldots,
$$

for all $t_{0}$ and $t_{1}$, even as $t_{0}, t_{1} \rightarrow \infty$.

Substituting this expansion into (5) and collecting like powers of $\varepsilon$ gives us equations for $p_{0}$ and $p_{1}$ as

$$
g^{\prime}\left(t_{1}\right) D_{0} p_{0}=r\left(t_{1}\right) p_{0} \ln \left(K\left(t_{1}\right)\right)-r\left(t_{1}\right) p_{0} \ln \left(p_{0}\right),
$$

and

$$
g^{\prime}\left(t_{1}\right) D_{0} p_{1}+D_{1} p_{0}=r\left(t_{1}\right) p_{1} \ln \left(K\left(t_{1}\right)\right)-r\left(t_{1}\right) p_{1} \ln \left(p_{0}\right)-r\left(t_{1}\right) p_{1} .
$$

respectively. Solving (8) gives

$$
p_{0}=K\left(t_{1}\right) \exp \left[-\gamma\left(t_{0}, t_{1}\right)\right], \quad \gamma\left(t_{0}, t_{1}\right)=\exp \left[-\frac{r\left(t_{1}\right)}{g^{\prime}\left(t_{1}\right)}\left(t_{0}+A\left(t_{1}\right)\right)\right],
$$

where $A\left(t_{1}\right)$ is an arbitrary function of $t_{1}$. Assuming that $A\left(t_{1}\right)$ and $g^{\prime}\left(t_{1}\right)$ are bounded positive functions of $t_{1}, p_{0}$ is bounded for all $t_{0}$ and $t_{1}$, and tends to $K\left(t_{1}\right)$ as $t_{0} \rightarrow \infty$. Equation (9) becomes, on rearrangement,

$$
D_{0} p_{1}+\frac{r\left(t_{1}\right) p_{1}}{g^{\prime}\left(t_{1}\right)}\left[1-\ln \left(\frac{K\left(t_{1}\right)}{p_{0}}\right)\right]=-\frac{1}{g^{\prime}\left(t_{1}\right)} D_{1} p_{0} .
$$

Note that $p_{0}$ contains one arbitrary function of $t_{1}$. Since the original equation (5) is first order, we seek a particular solution only of (11) to represent $p_{1}$. An integrating factor method gives

$$
p_{1}=\exp \left[-\frac{r\left(t_{1}\right)\left(t_{0}+A\left(t_{1}\right)\right)}{g^{\prime}\left(t_{1}\right)}-\gamma\left(t_{0}, t_{1}\right)\right] \times\left\{-\frac{K^{\prime}\left(t_{1}\right)}{r\left(t_{1}\right)} \exp \left[\frac{r\left(t_{1}\right) t_{0}}{g^{\prime}\left(t_{1}\right)}\right]\right.
$$




$$
\begin{aligned}
& -\frac{K\left(t_{1}\right) r^{\prime}\left(t_{1}\right) t_{0} \exp \left[\frac{-r\left(t_{1}\right) A\left(t_{1}\right)}{g^{\prime}\left(t_{1}\right)}\right]}{g^{\prime}\left(t_{1}\right)^{2}}\left(\frac{t_{0}}{2}+A\left(t_{1}\right)+\frac{A^{\prime}\left(t_{1}\right) r\left(t_{1}\right)}{r^{\prime}\left(t_{1}\right)}\right) \\
& \left.+\frac{K\left(t_{1}\right) r\left(t_{1}\right) t_{0} g^{\prime \prime}\left(t_{1}\right) \exp \left[\frac{-r\left(t_{1}\right) A\left(t_{1}\right)}{g^{\prime}\left(t_{1}\right)}\right]}{g^{\prime}\left(t_{1}\right)^{3}}\left(\frac{t_{0}}{2}+A\left(t_{1}\right)\right)\right\} .
\end{aligned}
$$

On substituting $p_{0}$ and $p_{1}$ into the Poincaré expansion (6) we obtain our two term approximation for the solution.

Under the assumptions above regarding $A\left(t_{1}\right)$ and $g^{\prime}\left(t_{1}\right), p_{1}$ involves terms such as $t_{0} \exp \left[-f\left(t_{1}\right) t_{0}\right]$ and $t_{0}^{2} \exp \left[-f\left(t_{1}\right) t_{0}\right]$, where $f\left(t_{1}\right)$ is a positive function. Thus, the ratio $p_{1} / p_{0}$ may become unbounded as $t_{0} \rightarrow \infty$, violating the condition (7). To overcome this we set the coefficients of these terms to zero, by choosing

$$
A^{\prime}\left(t_{1}\right)=0 \quad \Rightarrow \quad A\left(t_{1}\right)=C,
$$

where $C$ is an arbitrary constant, and

$$
g^{\prime}\left(t_{1}\right)=r\left(t_{1}\right) .
$$

This last condition gives the time scales $t_{0}$ and $t_{1}$ as

$$
t_{0}=\frac{1}{\varepsilon} \int_{0}^{t_{1}} r(s) d s \text { and } t_{1}=\varepsilon t .
$$

Note that when $r(\varepsilon t)$ is constant, these scales reduce to the simpler scales $t$ and $\varepsilon t$ as used in [7].

Substituting the above into the expansion (6), we obtain

$$
p\left(t_{0}, t_{1}, \varepsilon\right)=\left(K\left(t_{1}\right)-\frac{\varepsilon K^{\prime}\left(t_{1}\right)}{r\left(t_{1}\right)}\right) \exp \left[-C e^{-t_{0}}\right]+\cdots .
$$

Using the initial conditions that at $t=t_{0}=t_{1}=0, p=P_{0}$, and rewriting in the original variables, we obtain the expansion

$$
P(t, \varepsilon)=\left(K(\varepsilon t)-\frac{\varepsilon K^{\prime}(\varepsilon t)}{r(\varepsilon t)}+\frac{\varepsilon K^{\prime}(0) K(\varepsilon t)}{r(0) K(0)} \exp \left[-\frac{1}{\varepsilon} \int_{0}^{\varepsilon t} r(s) d s\right]\right) \times
$$




$$
\times\left(\frac{P_{0}}{K(0)}\right)^{\exp \left[-\frac{1}{\varepsilon} \int_{0}^{\varepsilon t} r(s) d s\right]}
$$

Equation (17) provides a two term approximation to the solution of the Gompertz model when the growth rate and carrying capacity vary slowly with time. As time, $t \rightarrow \infty$, the population, $P(t, \varepsilon)$, as approximated by $(17)$, tends to the carrying capacity, $K(\varepsilon t)$, minus an $O(\varepsilon)$ term dependent on the derivative of the carrying capacity $K^{\prime}(\varepsilon t)$ and the growth rate $r(\varepsilon t)$. Further, in many circumstances, the carrying capacity tends to a constant value as $t \rightarrow \infty$; that is, $K^{\prime}(\varepsilon t) \rightarrow 0$, so that $P(t, \varepsilon)$ given by $(17)$ actually tends to the carrying capacity, as in the constant $K$ case.

\section{Application to particular cases}

\subsection{Exponentially varying carrying capacity}

We consider a carrying capacity that either grows or decays exponentially and slowly over time:

$$
K(\varepsilon t)=K_{0} \exp [\varepsilon \rho t],
$$

where $\varepsilon$ is small, $K_{0}$ is the initial carrying capacity and $\rho$ is a constant . For $\rho>0, K$ increases exponentially, whereas for $\rho<0$, it decays. Such an exponential growth in a population could arise from an increase in the ability to produce more food or the introduction of vaccines into an environment. The growth rate $r$ is constant.

Figure 1 shows the results of applying the expansion (17), for values $K_{0}=200, \rho=0.2, P_{0}=20, r=0.2$ and $\varepsilon=0.1$. For comparison, the carrying capacity and the results of a numerical solution are included.

Equation (17) provides an accurate representation of the evolving population, that tends to values in a small neighbourhood of the carrying capacity. 


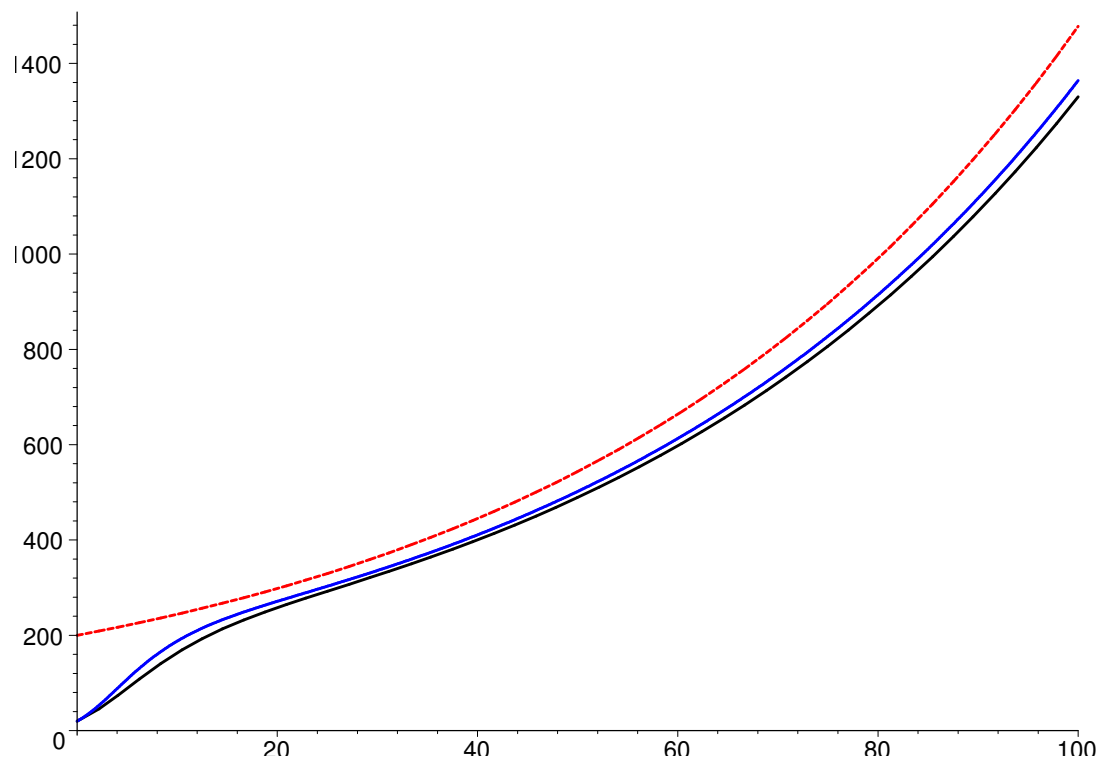

FiguRE 1: Multi-scale approximation (black) vs. numerical solution (blue) for exponentially varying carrying capacity (red). 
Here $K^{\prime}(\infty) \neq 0$, so that $P=K+O(\varepsilon)$ at best as $t \rightarrow \infty$. These features are reflected in the numerical solution.

\subsection{Periodically varying carrying capacity}

A periodically slowly varying carrying capacity may be

$$
K(\varepsilon t)=K_{0}+\delta \sin (\varepsilon t),
$$

where $\varepsilon$ is small. Here, $K$ executes slow oscillations about $K_{0}$ of amplitude $\delta$. Such behavior of the carrying capacity is a typical response to environments which fluctuate slowly over time. For example, in a marine environment the changing tides resulting from the phases of the moon bring slow variation in the ability of the environment to support a given species. Figure 2 compares the expansion (17) with the carrying capacity and the results of a numerical solution, for values $K_{0}=20, P_{0}=10, \delta=0.3$ and $\varepsilon=0.1$.

The population does not stay strictly below the carrying capacity, as in the previous case, but rather, remains in a small neighbourhood of it as time evolves. Since $K^{\prime}$ alternates in sign, the contribution of the second term of (17) alternates as well-hence the switching from above $K$ to below. As for the example above, this behaviour is reflected in the numerical solution.

\subsection{The bi-Gompertz model}

In some logistic evolving systems,the carrying capacity $K$ displays a logistic type growth between two constant limits [1]. Such a "pulse" in $K$ is reflected in the corresponding population. Here, we copy the approach in [1], to obtain a slowly varying "bi-Gompertz" form of $K$, varying between two constant limits. This is then applied to (17), to obtain a representation for 


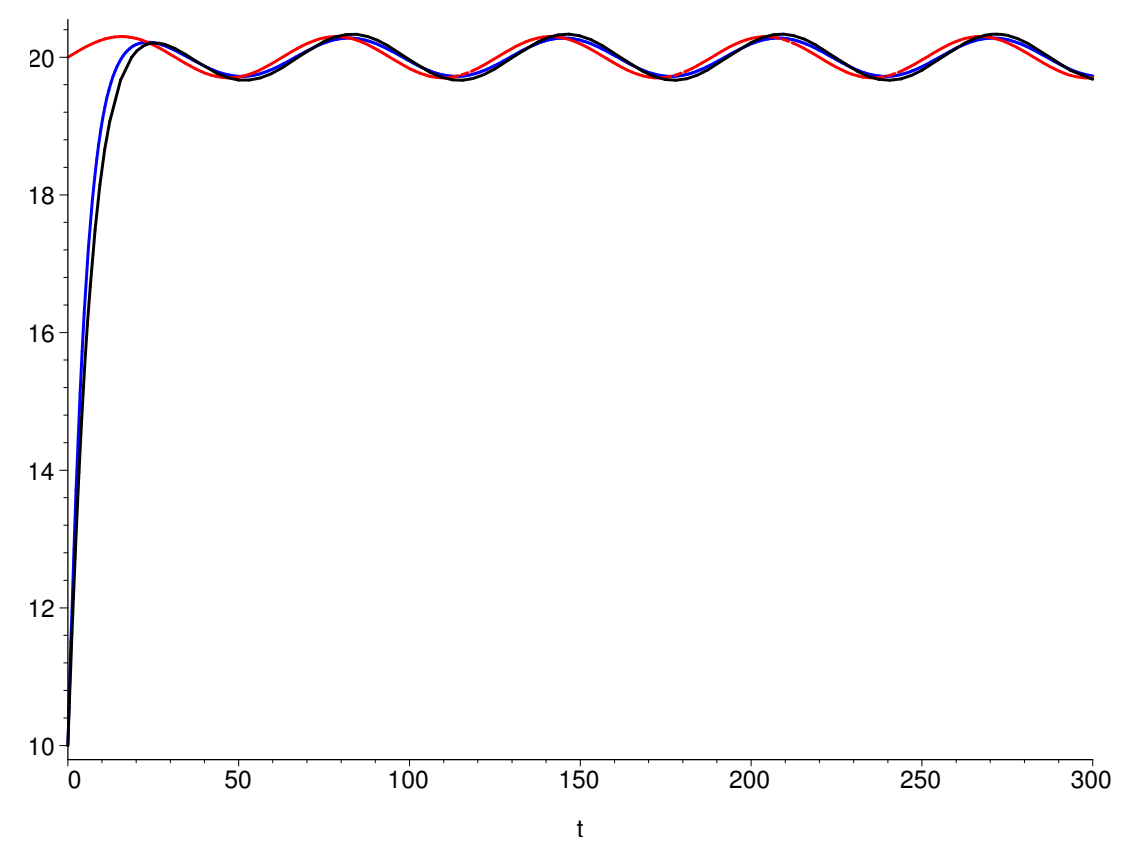

FIGURE 2: Multi-scale approximation (black) versus numerical solution(blue) for periodic carrying capacity (red). 


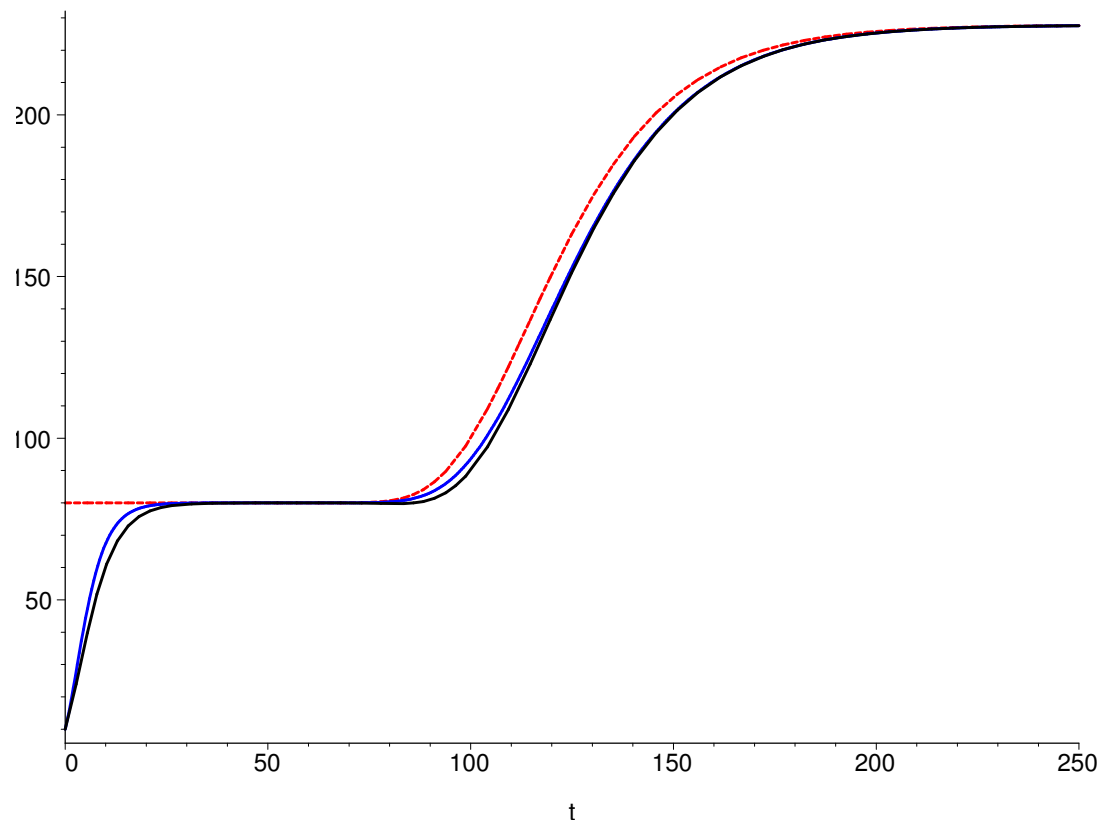

FigURE 3: Numerical solution (blue) versus multi-timing approximation (black) with carrying capacity (red).

the variation of the population. The appropriate form for $K$ is

$$
K(t)=k_{1}+k_{2} \exp \left[\frac{r}{\varepsilon}\left(1-e^{-\varepsilon\left(t-t_{m k}\right)}\right)\right],
$$

where $k_{1}$ is the initial carrying capacity, $r$ and $\varepsilon$ are constant growth rates for the first and second growth phases, and $t_{m k}$ is the location of the second turning point in time. Figure 3 shows the results of this for $k_{1}=K_{0}=80$, $k_{2}=20, \varepsilon=0.05, t_{m k}=100, P_{0}=10$ and $r=0.1$.

Once again the approximation is acceptably accurate as there is minimal difference between the numerical solution and the two term multi-timing approximate solution. 


\subsection{Periodically varying growth rate and carrying capacity}

The preceding examples only display a varying carrying capacity. Clearly (17) allows for $r$ to vary (slowly) as well. Here, we consider growth rates and carrying capacities that are varying slowly and periodically with time, since these may arise in environments which fluctuate slowly over time.

Representative functions for such $r(\varepsilon t)$ and $K(\varepsilon t)$ are

$$
\begin{aligned}
& r(\varepsilon t)=r_{0}+\delta \sin (\varepsilon t), \\
& K(\varepsilon t)=K_{0}+\Delta \sin (\varepsilon t),
\end{aligned}
$$

where $r_{0}$ and $K_{0}$ are mean values and $\delta$ and $\Delta$ denote amplitudes of the respective oscillations about these means.

Figure 4 displays the results obtained using (17) where $r_{0}=0.5, \delta=0.14$, $K_{0}=50$ and $\varepsilon=0.1$. The numerical (the blue line) and approximate solutions (the black line) are effectively coincident, while both tend rapidly to the carrying capacity (red line) as time progresses.

\section{Discussion}

The calculations above have yielded a useful approximation to the solutions of the Gompertz model, valid for arbitrary slowly varying growth rate $r$ and carrying capacity $K$. This agrees very well with the results of numerical computations and has the further advantage of being an explicit function, capable of representing general features of the evolving population. Numerical solutions can only achieve this at the expense of numerous recalculations using very specific data.

While these methods have been applied here to a particular population model, they are readily applicable, with suitable modifications, to a wide 


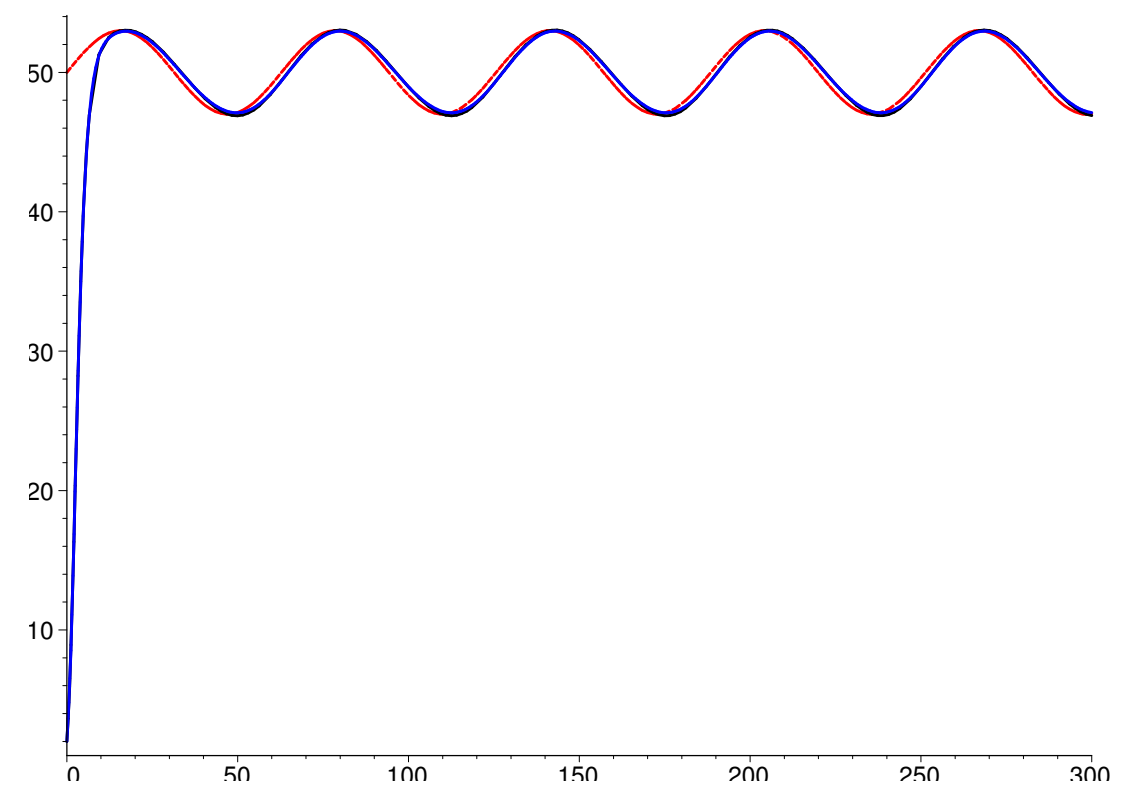

Figure 4: Periodically varying $r$ and $K$. 
range of single-species models. As noted earlier, a simpler version of the two time scales used here has also been applied to a logistic model with slowly varying carrying capacity [7] . Similarly, extension to a harvesting model has also been investigated [5].

\section{References}

[1] Ausubel, J. H. et al., Carrying capacity: a model with logistically varying limits, Technological Forecasting and Social Change, 61(3), pp. 209-214, 1999. doi:10.1016/S0040-1625(99)00022-0 C549

[2] Banks, R. B., Growth and Diffusion Phenomena: Mathematical Frameworks and Application, Springer-Verlag, Berlin, Germany, 1994. C543

[3] Gompertz, B., On the nature of the function expressive of the law of human mortality, and on a new mode of determining the value of life contingencies, Phil. Trans. Roy. Soc. London, 123, pp. 513-585. 1825. C543

[4] Grozdanovski, T., Slow Variation in the Single Species Gompertz Population Model, Honours Thesis, School of Mathematical and Geospatial Science, RMIT University, 2005. C543

[5] Grozdanovski, T., Nguyen, L. and Shepherd, J. J., A slowly varying harvesting model, in preparation. C554

[6] Murdock, J. A., Perturbations Theory and Methods, Society for Industrial and Applied Mathematics, USA, 1999. C543

[7] Stojkov, L. Population Modelling with Slowly Varying Carrying Capacities, Honours Thesis, Mathematics Department, RMIT University, 2003. C544, C546, C554 